\title{
Publisher Correction: Doping-induced disappearance of ice II from water's phase diagram
}

\author{
Jacob J. Shephard, Ben Slater, Peter Harvey, Martin Hart, Craig L. Bull, Steven T. Bramwell and Christoph G. Salzmann (1)
}

Correction to: Nature Physics https://doi.org/10.1038/s41567-018-0094-z, published online 2 April 2018.

In the version of this Letter originally published, the citation to ref. 30 in the Fig. 1 caption should have been to ref. 29, and the citation to ref. 29 in the Methods should have been to ref. 30 .

Published online: 11 April 2018

https://doi.org/10.1038/s41567-018-0135-7

\section{Publisher Correction: Geometric constraints during epithelial jamming}

Lior Atia, Dapeng Bi iD, Yasha Sharma, Jennifer A. Mitchel, Bomi Gweon, Stephan A. Koehler, Stephen J. DeCamp (iD, Bo Lan, Jae Hun Kim, Rebecca Hirsch, Adrian F. Pegoraro, Kyu Ha Lee, Jacqueline R. Starr, David A. Weitz, Adam C. Martin, Jin-Ah Park, James P. Butler and Jeffrey J. Fredberg

Correction to: Nature Physics https://doi.org/10.1038/s41567-018-0089-9, published online 2 April 2018.

In the version of this Article originally published, the Supplementary Movies were linked to the wrong descriptions. These have now been corrected. Additionally, the authors would like to note that co-authors James P. Butler and Jeffrey J. Fredberg contributed equally to this Article; this change has now been made.

Published online: 19 April 2018

https://doi.org/10.1038/s41567-018-0139-3

\section{Author Correction: Geometric constraints during epithelial jamming}

Lior Atia, Dapeng Bi ID, Yasha Sharma, Jennifer A. Mitchel, Bomi Gweon, Stephan A. Koehler, Stephen J. DeCamp (D, Bo Lan, Jae Hun Kim, Rebecca Hirsch, Adrian F. Pegoraro, Kyu Ha Lee, Jacqueline R. Starr, David A. Weitz,

Adam C. Martin, Jin-Ah Park, James P. Butler and Jeffrey J. Fredberg

Correction to: Nature Physics https://doi.org/10.1038/s41567-018-0089-9, published online 2 April 2018; corrected online 19 April 2018. In the first correction to this Article, the authors added James P. Butler and Jeffrey J. Fredburg as equally contributing authors. However, this was in error; the statement should have remained indicating that Lior Atia, Dapeng Bi and Yasha Sharma contributed equally. This has now been corrected. 